\title{
Sliding Mode Current Controller Design for Power LEDs
}

\author{
Eung-Seok Kim* and Cherl-Jin Kim ${ }^{\dagger}$
}

\begin{abstract}
High-brightness LED control is required for stable operation, thus the driver and control system must be designed to deliver a constant current to optimize reliability and ensure consistent luminous flux. In this paper, the sliding mode current controller is designed to adjust the illumination density of power LEDs. The controller design model of power LEDs, including its driving circuit, is proposed to realize the dimming control of power LEDs. A buck converter is introduced to drive the power LEDs and reduce the input voltage to a lower level. The sliding mode software controller is implemented to adjust the dimming of power LEDs. The proposed strategy for driving power LEDs is investigated and comparatively studied by experiments.
\end{abstract}

Keywords: Power LED, Dimming control, Buck converter, Sliding mode control

\section{Introduction}

Due to increased carbon dioxide emissions, climate change has occurred, and this has been a serious problem being faced by human beings. Recently, there is an increase prevalence of LEDs for electrical energy saving and reduction of carbon dioxide emission. LED is a solid-state semiconductor device that directly converts electrical energy into light. It has many advantages such as having long life, good energy efficiency, and energy saving. In addition, the field and market for the application of LED is rapidly expanding because of the control flexibility of lighting and light intensity. LED is a light source of semiconductor; the forward voltage drop is over 2.5 [V]; and thus the excessive heat generation is an inevitable disadvantage. For these reasons, the current and brightness of LED are varied depending on temperature variation by the constant voltage control method. However, when the constant voltage control method is used for power LED driving, LEDs are damaged. Therefore, high-power LEDs require precise control over the current to maintain efficient and reliable output color wavelength of the LED. A switching regulator regulates the current flow by switching the input voltage and controlling the average current by means of the duty cycle or on time. If a higher current is required, the percentage of being on time is increased to accommodate the load change. In [1], it is represented for three considerations of a current mode control such as peak, valley, and emulated control. The current mode control with respect to the peak is presented in [2]. A new continuous-time model for current mode control is proposed in [3]. A unified model for current-programmed converters is proposed in [4]. According to studies on the current mode control to date, it is represented for three considerations of a current mode control

\footnotetext{
$\dagger \quad$ Corresponding Author: Department of Electrical and Electronic Engineering, Halla University, Wonju, Korea. (cjkim@halla.ac.kr)

* Department of Control and Instrumentation Engineering, Halla University, Wonju, Korea. (eskim@halla.ac.kr)

Received: June 7, 2010; Accepted: November 2, 2010
}

such as peak, valley, and emulated control [1], [8], [9]-[11]. Particularly, the current mode control with respect to the peak current is presented by R. Seehan, and a new continuous-time model for current mode control is studied by Ridley et al. [2], [3]. A current programmable unified model of the converter has been proposed previously; this model has advantages to be amended and scaled to similar model [4].

In this paper, we present the design method of buck-type converter to regulate the driving current of power LEDs. We construct the mathematical model for the parameter uncertainties of power LEDs and its drive circuit and design the current controller to adjust the light intensity of illumination of power LEDs. The proposed controller consists of microcontroller (MCU) and analog circuits. MCU is used to generate the PWM signal for regulating the driving current of power LEDs, and this has several advantages such as simple implementation of PWM dimming control of power LEDs. This paper consists of some sections. In section 2, we present the controller design model for the parameter uncertainty of the power LEDs with its drive circuits using buck-type converter. In section 3, we study the design problem of the sliding mode current controller. In section 4, we investigate the validity of the proposed control system for the driving circuits of power LEDs by using an experimental setup. We collate the concluding remarks in section 5 .

\section{Controller Design Model of Power LEDs and Its Driving Circuits}

In forward-bias mode, the $V-I$ and $V-R$ characteristic curves of the power LED are nonlinear, and its impedance $R_{L}\left(=Z_{L E D}=V / I\right)$ is proportional to the magnitude of the voltage $(V)$ and the current $(I)$. Since the voltage and the current of the LED have nonlinear characteristics, the decreasing efficiency of the LED is very clear. LED is a light 
source of semiconductor; the forward voltage drop is over $2.5[\mathrm{~V}]$; and thus the excessive heat generation is an inevitable disadvantage. For these reasons, the current and brightness of LED are varied depending on temperature variation by the constant voltage control method. However, when this method is used for power LED driving, LEDs are damaged. Therefore, the constant current control method is required for driving power LED. To design the constant current controller of power LEDs, we must consider its nonlinear impedance characteristic. The impedance of power LED $Z_{L E D}$ can be regarded as a timevarying parameter since it is irregularly variable as time elapses after the circuit power is turned on. The impedance of power LED is dependent on the voltage and the current, thus we design the current controller considering this impedance characteristic to enhance the illumination efficiency of the power LEDs. In order to control the driving current of power LEDs, buck-type converter is selected. Since the buck-type DC/DC converter operates in an ON/OFF switching mode, its dynamic model is very complicated. Therefore, we used the circuit averaging method to construct the mathematical model of the converter. In Fig. 1, we represent the equivalent circuit of the buck-type converter. In this circuit, the input voltage $V_{D C}$ is the DClink voltage and the output voltage $V_{C}$ is the capacitor. In Fig. 1, $\mathrm{R}_{\mathrm{S}}$ is the resistor for a current measurement and $Z_{L E D}$ is the equivalent impedance of the LED. For simplicity, we regard the equivalent resistances of $L$ and $C$ as $R_{S}$ and $Z_{L E D}$ including those.

Choosing the connecting points of switch $S_{1}$ and the diode as the nodes a, p, and c, respectively, we can obtain the current eq. (1) and the voltage eq. (2) as follows:

$$
\begin{aligned}
& i_{a}=\left\{\begin{array}{ccc}
\left(i_{c}(t)\right. & \text { if } & S_{1} \text { ON } \\
0 & \text { else } & S_{1} \text { OFF }
\end{array}\right. \\
& v_{c p}=\left\{\begin{array}{ccc}
v_{a p}(t) & \text { if } & S_{1} \text { ON } \\
0 & \text { else } & S_{1} \text { OFF }
\end{array}\right.
\end{aligned}
$$

Applying the duty ratio $D$ of the switch to eqs. (1) and (2), we can obtain eqs. (3) and (4) as the average of a period, as expressed below:

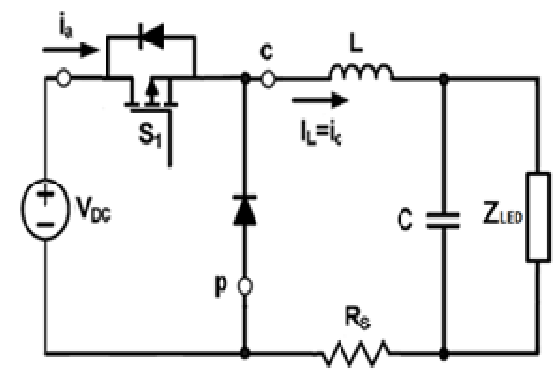

Fig. 1. Power LED drive circuit using buck-type converter.

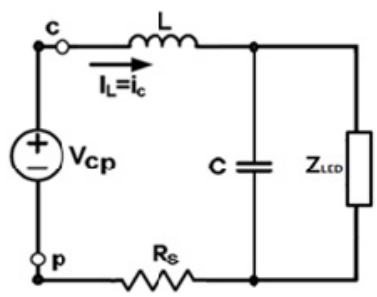

Fig. 2. Equivalent circuit of the power LED drive.

$$
\begin{aligned}
& i_{a}=D i_{c} \\
& v_{c p}=D v_{a p}
\end{aligned}
$$

Using eq. (4) with respect to $v_{c p}$ as the input voltage, we can redraw the circuit in Fig. 1 as follows:

In the above circuit, we can regard $v_{c p}$ as the average voltage applied to the circuit [1]. Using the differential operator, we can represent the input voltage $v_{c p}$ and the output current $i_{L}$ as follows:

$$
\begin{aligned}
\frac{i_{L}}{v_{c p}} & =\frac{1}{z_{L}+z_{C R}} \\
Z_{L} & =s L+R_{S} \\
Z_{C R} & =\frac{z_{L E D}}{1+s C Z_{L E D}}
\end{aligned}
$$

Where " $s$ " is the differential operator. Rewriting eq. (5) using eqs. (6) and (7), we can obtain the relationship of the output current and the input voltage as follows:

$$
\frac{i_{L}}{v_{c p}}=\frac{s C Z_{L E D}+1}{s^{2} L C Z_{L E D}+s\left(L+C Z_{L E D} R_{S}\right)+R_{S}+Z_{L E D}}
$$

Since the $Z_{L E D}$ is nonlinear, the differential equation of eq. (8) is as follows:

$$
\begin{aligned}
& L C\left[Z_{L E D} \ddot{y}+2 \dot{Z}_{L E D} \dot{y}+\ddot{Z}_{L E D} y\right] \\
& +\left[\left(L+C Z_{L E D} R_{S}\right) \dot{y}+C R_{S} \dot{Z}_{L E D} y\right] \\
& +\left[\left(R_{S}+Z_{L E D}\right) y\right] \\
& =\left[C Z_{L E D} \dot{u}+C \dot{Z}_{L E D} u\right]+u
\end{aligned}
$$

Where $y=i_{L}$, and $u=v_{c p}$. The controller design model expressed in eq. (9) is very complicated. If the capacitor $C$ in eq. (9) is negligibly small, we can overcome this complicated problem. The buck topology allows the power LED to be in series with the inductor at all times and limits the current ripple. Therefore, an output capacitor is not necessary as it would be for a current regulator circuit. However, an output capacitor can optionally be used to 
lower the required value of the inductor. Ignoring the effect of the output capacitor, i.e., $C=0$, we obtained the reduced order model of eq. (9) as follows:

$$
\dot{y}=-b_{0} f_{z} y+b_{0} u,
$$

Where $b_{0}=\frac{1}{L}, f_{z}\left(=R_{S}+Z_{L E D}\right)$ is nonlinear, although we do not need to know this exactly.

\section{Current Controller Design}

In this section, we deal with the current control problem using the first-order system represented in eq. (10). For this system, we designed the sliding mode controller as follows :

$$
\begin{gathered}
u=\frac{1}{b_{0}}\left\{\dot{y}_{r}-a_{r} e+\bar{f}_{z} y-\beta \xi\right\} \\
\beta=\alpha_{1}\left|y_{\mathrm{r}}\right|+\alpha_{2}|u| \\
\xi=\operatorname{sign}(e)=\left\{\begin{array}{cc}
1, & e>0 \\
-1, & e<0
\end{array}\right\}
\end{gathered}
$$

Where $e\left(=y-y_{r}\right)$ is the output error and $\alpha_{1}$ and $\alpha_{2}$ are the design parameters. In addition, $\bar{f}_{z}$ and $\bar{b}_{0}$ are the known nominal values, while $\tilde{f}_{z}\left(=\bar{f}_{z}-f_{z}\right)$ and $\tilde{b}_{0}\left(=\bar{b}_{0}-b_{0}\right)$ are the parameter errors. We also designed the desired input as follows:

$$
\dot{y}_{r}=-a_{r} y_{r}+b_{r} r
$$

Where $r$ is the reference input and $a_{r}$ and $b_{r}$ are the positive constants, which serve as the design parameters.

Theorem: If there exist positive constants $\alpha_{1}, \alpha_{2}$ satisfying the inequalities of $\alpha_{1}>\left|\tilde{f}_{z}\right|, \bar{b}_{0}>\alpha_{2}>\left|\tilde{b}_{0}\right|$, then the system eq. (10) with the controller in eqs. (11)-(14) is stable; the output errors $e$ and $\dot{e}$ of the system converge to "0" exponentially fast; and all states of the system including the control input are bounded.

Proof: Substituting the controller eq. (11) into the system eq. (10), we obtained the closed loop system as follows:

$$
\dot{y}=-f_{z} y+\frac{b_{0}}{\bar{b}_{0}}\left\{\dot{y}_{r}-a_{r} e+\bar{f}_{z} y-\beta \xi\right\}
$$

Adding $\bar{b}_{0}-\bar{b}_{0}$ to the numerator term in eq. (15), we can rewrite it as follows :

$$
\begin{aligned}
\dot{y} & =-f_{z} y+\frac{\bar{b}_{0}+b_{\mathrm{o}}-\bar{b}_{0}}{\bar{b}_{0}}\left\{\dot{y}_{r}-a_{r} e+\bar{f}_{z} y-\beta \xi\right\} \\
& =\dot{y}-a_{r} e+\tilde{f}_{z} y-\tilde{b}_{0} u-\beta \xi
\end{aligned}
$$

Arranging eq. (16) with the output error $e\left(=y-y_{r}\right)$, we obtained the error equation as follows :

$$
\dot{e}=-a_{r} e+\tilde{\vartheta}^{\mathrm{T}} \omega-\beta \xi
$$

Where $\tilde{\theta}=\left[\begin{array}{ll}\tilde{f}_{\mathrm{z}} & \tilde{b}_{0}\end{array}\right]$ and $\omega=\left[\begin{array}{ll}y & -u\end{array}\right]$. Defining the Lyapunov function candidate as $W=\frac{1}{2} e^{2}$, its first time derivative is as follows:

$$
\dot{W}=-a_{r} e^{2}+\tilde{\vartheta}^{\mathrm{T}} \omega e-\beta \xi e
$$

Using eqs. (12) and (13), we obtained the inequality as follows:

$$
\begin{aligned}
\dot{W} & \leq-a_{r} e^{2}-\left\{\beta-\left|\tilde{\vartheta}^{\mathrm{T}} \omega\right|\right\}|e| \\
& \leq-a_{r} e^{2}-\left\{\alpha_{1}-\left|\tilde{f}_{\mathrm{z}}\right|\right\}|y||e|-\left\{\alpha_{2}-\left|\tilde{b}_{0}\right|\right\}|u \| e| \\
& \leq-a_{r} e^{2}=-2 a_{r} W
\end{aligned}
$$

Thus, the output error $e\left(=y-y_{r}\right)$ and $\dot{e}$ converge to " 0 " exponentially fast. We also showed that the control input as in eq. (11) satisfy the inequality as follows :

$$
|u| \leq \frac{1}{\bar{b}_{0}}\left\{\left|\dot{y}_{\mathrm{r}}\right|+a_{r}|e|+\left(\bar{f}_{z}+\alpha_{1}\right)|y|+\alpha_{2}|u|\right\}
$$

Since $0<\frac{\alpha_{2}}{\bar{b}_{0}}<1$ and $\left|\dot{y}_{r}\right|,|e|,|y|$ are bounded, we rewrite eq. (20) as follows :

$$
|u| \leq \alpha_{3}+\alpha_{4}|u|
$$

Where $\quad \alpha_{3} \geq \frac{1}{\bar{b}_{0}}\left(\left|\dot{y}_{r}\right|+a_{r}|e|+\bar{f}_{z}|y|+\alpha_{1}|y|\right) \quad$ and $0<\alpha_{4}\left(=\frac{\alpha_{2}}{b_{0}}\right)<1$. Therefore, the control input is also bounded, and we get the concluding remarks that all states of the proposed system are bounded and the error system is exponentially stable.

Remark: From the above results, choosing the parameters $\alpha_{r}$ as large as possible improves the exponential convergence and the system response.

\section{Numerical example}

For the variable step reference $r$, the output current $y\left(=i_{L}\right)$ of the power LED follows the reference 
$r(=200 \sim 350 m A)$, and the steady-state error of the above system converges to zero. The parameter variation does not affect the steady-state response. The design parameters are $\alpha_{r}=20, b_{\mathrm{r}}=20$, while the system parameters are $L=300[\mu H], C=0[F]$. The simulation results are represented in Figs. 3 and 4.

In the above results, the output current follows well the desired current, with the input voltage being bounded. The larger the desired output parameters $\alpha_{r}$ and $b_{r}$, the faster the output response that will be obtained.

\section{Experimental Setup and Results}

In this section, we represent the experimental setup as shown in Figs. 5 and 6. In order to drive the power LEDs, we used the buck-type converter and the FET switch in the circuit. To calculate the sliding mode control algorithm, we selected the 8-bit MCU using the output current measurement. The MCU used in the experiment can sufficiently calculate the duty ratio of the PWM pulse applied to the FET switch. The 8-bit MCU has 10-bit PWM resolution. The schematic of the proposed system is shown in Fig. 5, which has the same equivalent circuit as that in Fig. 1. Therefore, the overall system, as shown in Fig. 5, used 5 $[V]$ switching signal generated in the 8-bit MCU to control the FET switch. Because of the location of the sense resistor in the circuit, the current feedback signal is present only when the MOSFET is turned on and energy charging the inductor. Since the feedback signal is discontinuous, a simple peak hold circuit with a Shottky diode was used at the output of the current sense amplifier. The component values of the peak hold circuit were chosen to provide a low pass filter with a very low cutoff frequency, which averages the current feedback signal. To ensure that the current can be properly regulated using the software sliding mode controller, the cutoff frequency was chosen to be $1 / 10$ th of the value of the current sample rate [5]-[7].

The nominal values of the system states and their parameters are shown in Table 1 . The features of the power LED used in the experiments are represented in Table 2.

We have changed the LED current reference from 360

Table 1. The nominal states and parameters of the system

\begin{tabular}{c|cc}
\hline States and parameters & \multicolumn{2}{|c}{ Values } \\
\hline Input voltage, $V_{D C}$ & 12.00 & {$[V]$} \\
\hline Switching frequency, $f_{S W}$ & 125.00 & {$[\mathrm{kHz}]$} \\
\hline$L$ & 300.00 & {$[\mu \mathrm{H}]$} \\
\hline$C$ & 0.00 & {$[\mu \mathrm{F}]$} \\
\hline$R_{S}$ & 0.41 & {$[\Omega]$} \\
\hline
\end{tabular}

Table 2. Features of the power LED

\begin{tabular}{c|c}
\hline Typical luminous flux & $1051 \mathrm{~m}$ at $350 \mathrm{~mA}$ \\
\hline Optical efficiency & $93 \mathrm{~lm} / \mathrm{W}$ \\
\hline
\end{tabular}

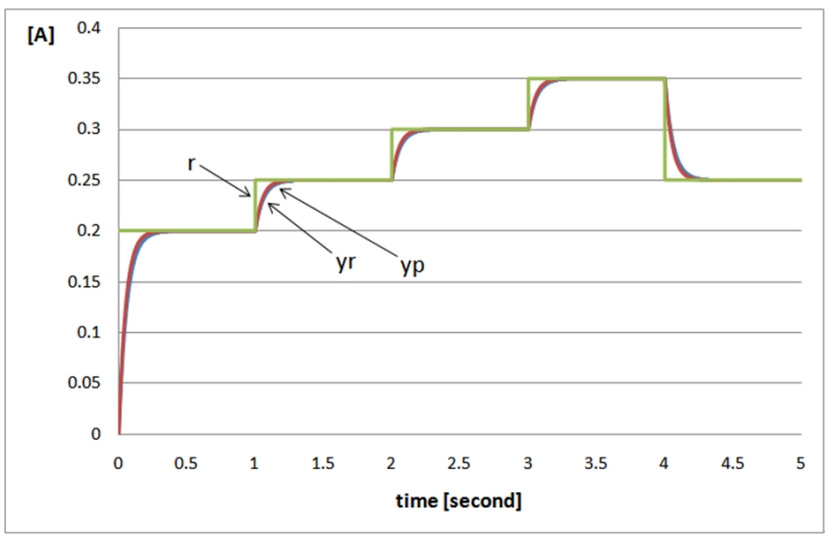

Fig. 3. Average output current.

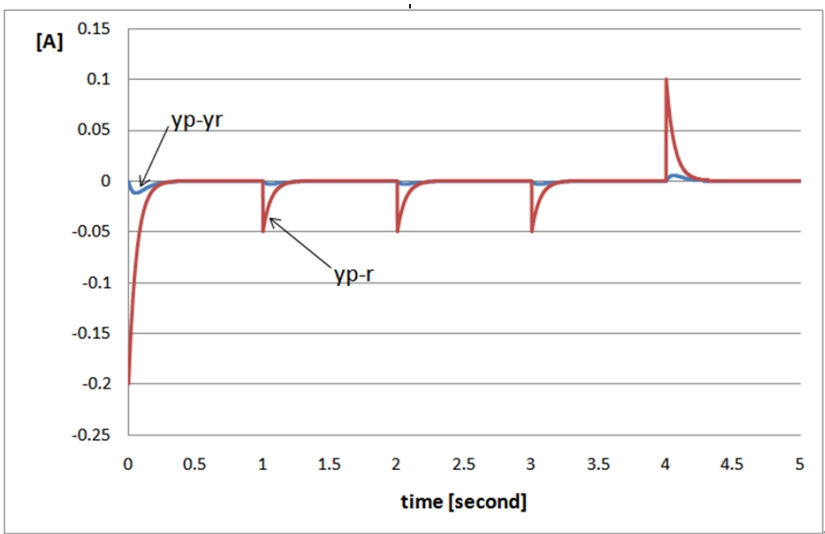

Fig. 4. Output errors.

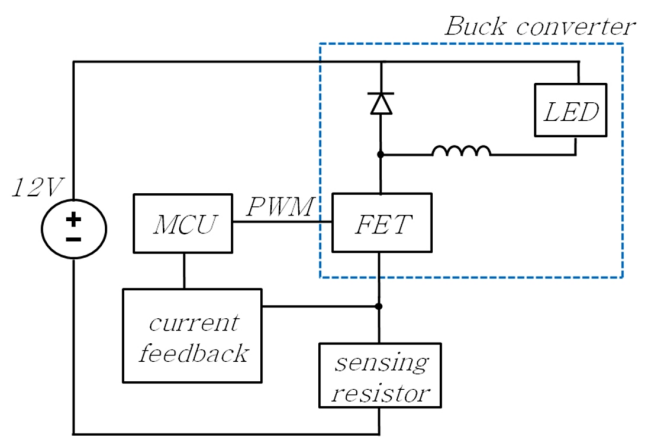

Fig. 5. Block diagram of overall system.

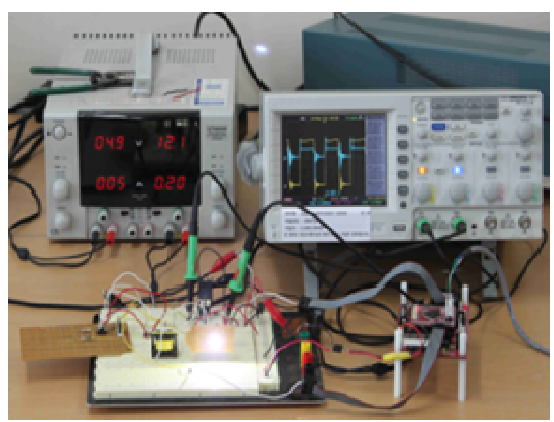

Fig. 6. Experimental setup for power LED drive. 
$\mathrm{mA}$ to $215 \mathrm{~mA}$, as shown in Fig. 7. The maximum duty ratio was restricted to $70 \%$ at $420[\mathrm{~mA}]$ for its marginal flexibility. As already mentioned, the sense resistor voltage of the current feedback signal is discontinuous as shown in Figs. 7 and 8. In order to obtain the continuous current feedback, a simple peak hold circuit with a Shottky diode was used at the output of the current sense amplifier. This continuous voltage of the current feedback was fed into the $\mathrm{ADC}$ of the MCU; the ADC value was directly used to calculate the control algorithm with the reference ADC value.

To show the validity of the proposed strategy, we performed an experiment comparing the sliding mode controller with the PI controller. In Figs. 7 and 8, we represent the waveforms of the sliding mode control and the PI control, respectively. We designed the proportional gain of the PI controller to be 35 and its integral gain to be 1 . We also selected the design parameters of the sliding mode controller as $\alpha_{r}=20, b_{\mathrm{r}}=20, \alpha_{1}=10$, and $\alpha_{2}=0.01$. Comparing Fig. 7 with Fig. 8, it can be seen that the PI controller requires more energy and has slower response time than the sliding mode controller. The output current of the sliding mode controller is also in good agreement with the current reference compared with the PI controller, as shown in Fig. 9. The output current error of each controller is shown in Fig. 10.

From the results, we can conclude that the current control performed well using the software sliding mode controller. However, it is necessary to reduce the oscillation of the sense signal caused by the switching operation.

\section{Conclusion}

In this paper, we studied the dimming control and the current regulating problems of power LEDs. We have designed the current driving circuit and the software current controller with consideration of the nonlinear characteristic of power LEDs. We also introduced the mathematically reduced order model to design the current controller. We used a buck-type converter to implement the current driving circuit of power LEDs. The overall system was con-

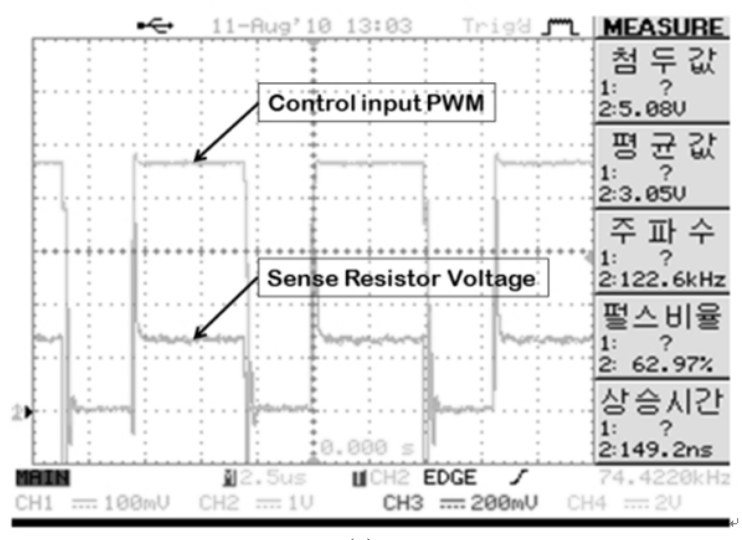

(a)

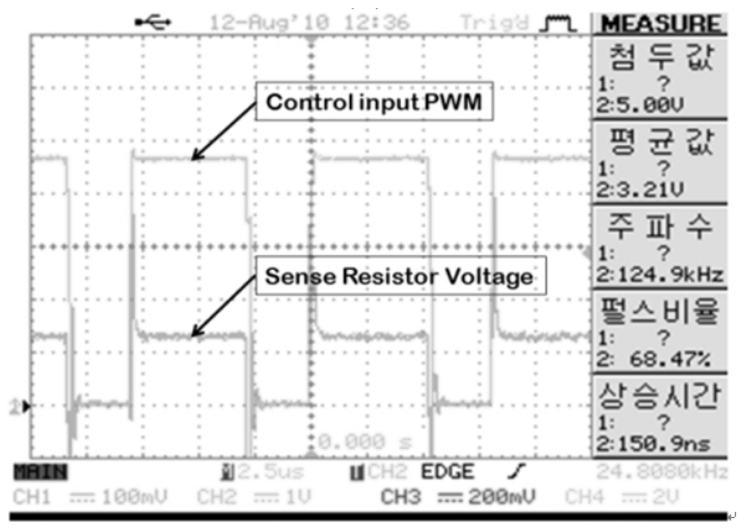

(b)

Fig. 7. The control input PWM signal and the sense resistor voltage at the ref. current of 360 [mA]: (a) sliding mode control and (b) PI control.

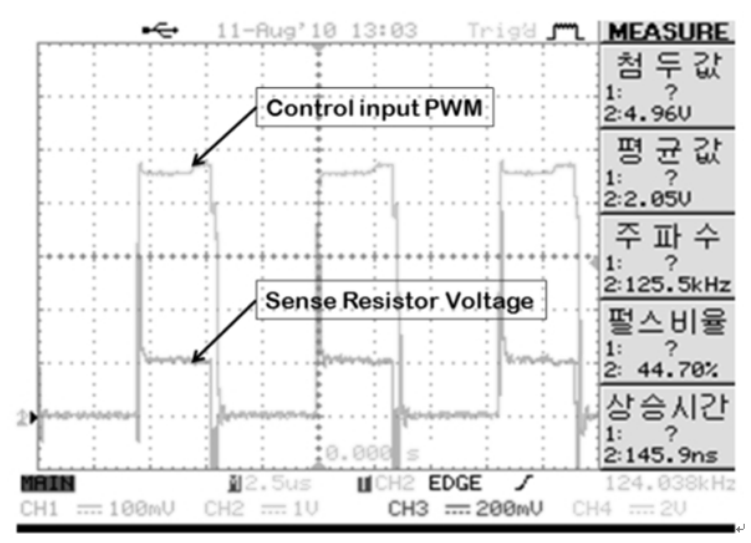

(a)

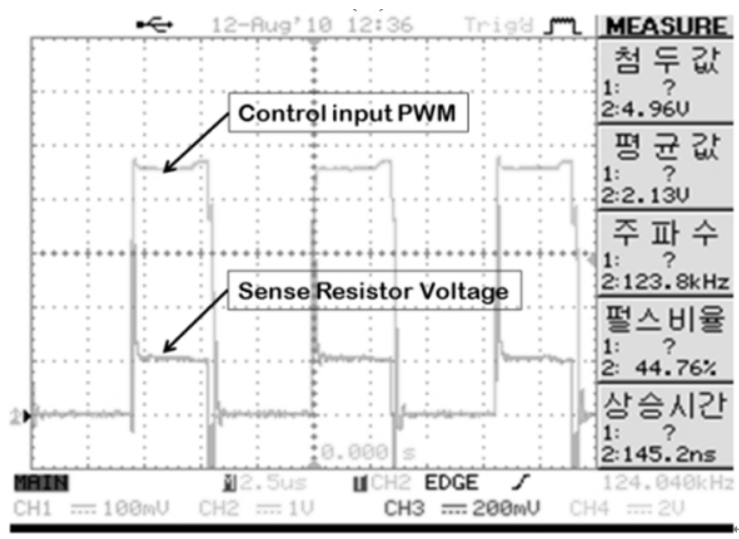

(b)

Fig. 8. The control input PWM signal and the sense resistor voltage at the ref. current of 215[mA]: (a) sliding mode control and (b) PI control. 


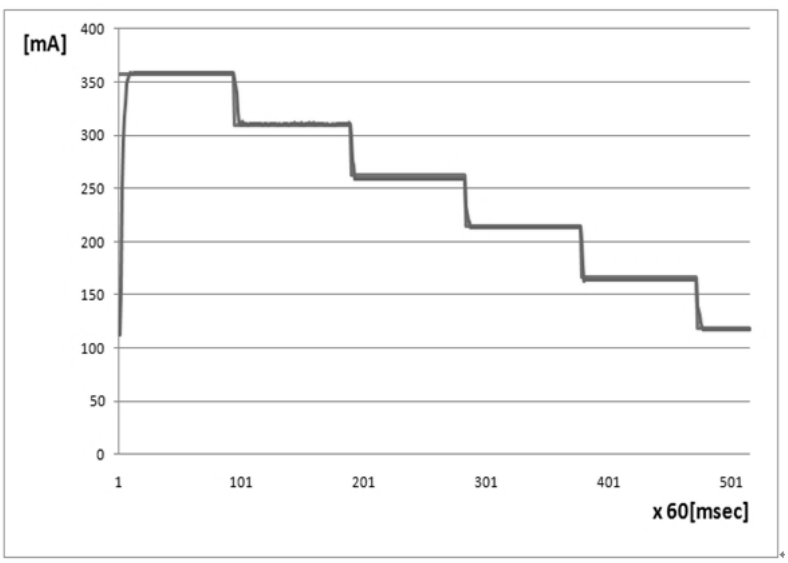

(a)

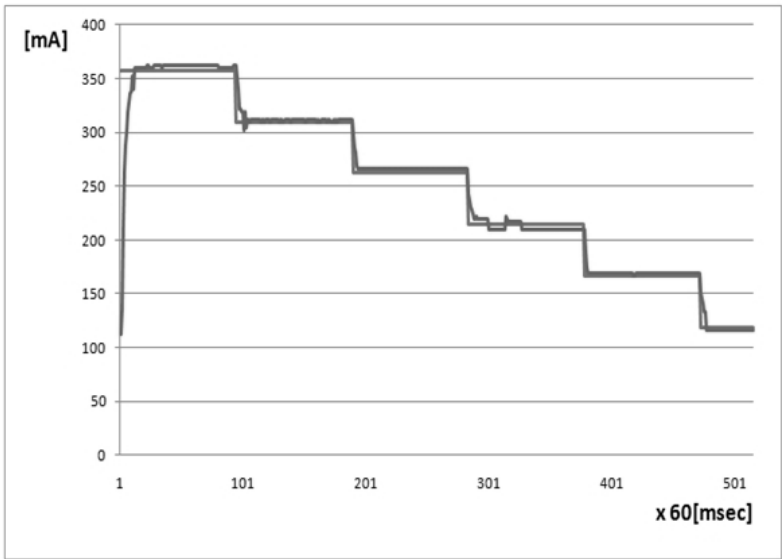

(b)

Fig. 9. The output current and the reference: (a) sliding mode control and (b) PI control.

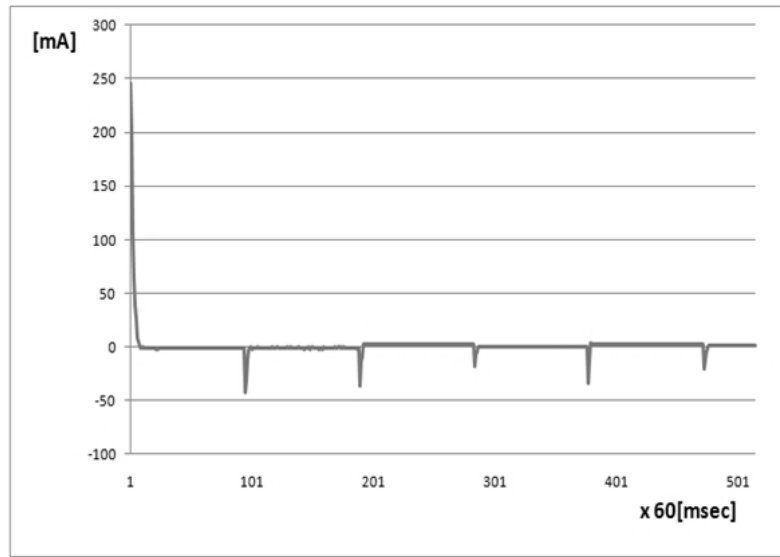

(a)

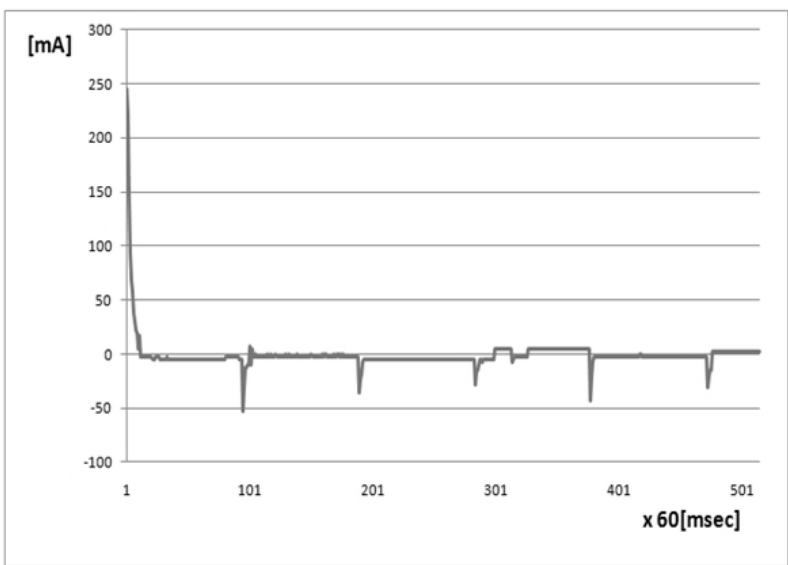

(b)

Fig. 10. The output current error: (a) sliding mode control and (b) PI control.

structed with two parts - the microcontroller for software dimming control and the analog circuit for the power LEDs current driving. We have shown from the experimental results that the power LEDs current is in good agreement with the reference with step-wise varying. Research to address the robustness issues for the current control system in the presence of bounded disturbances and uncertainties is currently ongoing.

\section{References}

[1] R. Sheehan, "Current-Mode Modeling for Peak, Valley and Emulated Control Methods - Reference Guide for Fixed-Frequency, Continuous ConductionMode Operation," National Semiconductor, http:// www.techonline.com, July, 2007.

[2] Sheehan, Robert, "Emulated Current Mode Control for Buck Regulators Using Sample and Hold Technique," Power Electronics Technology Exhibition and Conference, PES02, October, 2006.
[3] Ridley, R.B., “A New, Continuous-Time Model for Current Mode Control," IEEE Transactions on Power Electronics, Vol. 6, Issue 2, pp. 271-280, 1991.

[4] Tan, F.D. and Middlebrook, R.D., "A Unified Model for Current-Programmed Converters," IEEE Transactions on Power Electronics, Vol. 10, Issue 4, pp. 397 408, 1995.

[5] Stephen Bowling, "A Digital Constant Current Power LED Driver," Microchip Technology Inc. 2007.

[6] C. J. Kim, N. J. Choi, H. J. Lee, E. S. Kim, “A Digital LED Controller design with Constant Current," KIEE Proceeding, CD PEP 34, Jul., 2009.

[7] E. S. Kim, C. J. Kim, "A Constant Current Controller Design for Power LED Drive," Transactions on KIEE, Vol. 59, No. 3, 2010.

[8] Anbukumar Kavithar, Govidarajan Uma, "Resonant Parametric Perturbation method to Control Chaos in Current Mode Controlled DC-DC Buck-Boost Converter," Journal of Electrical Engineering \& Technology Vol. 5, No. 1, pp. 171-178, 2010.

[9] Alireza Khaligh and Ali Emadi, " Stabilizing Control 
of DC/DC Buck Converters with Constant Power Loads in Continuous Conduction and Discontinuous Conduction Modes Using Digital Power Alignment Technique," Journal of Electrical Engineering \& Technology, Vol. 1, No. 1, pp. 63-72, 2006.

[10] Tuyen D. Nguyen, Hong-Hee Lee and Hoang M. Nguyen, "Adaptive Carrier-based PWM for a FourSwitch Three-Phase Inverter under DC-link Voltage Ripple Conditions," Journal of Electrical Engineering \& Technology Vol. 5, No. 2, pp. 290-298, 2010.

[11] Ehsan Rokhsat-Yazdi, Ali Afzali-Kusha, and Massoud Pedram, "A High-Efficiency, Auto Mode-Hop, Variable-Voltage, Ripple Control Buck Converter," Journal of Power Electronics, Vol. 10, No. 2, pp. 115$124,2010$.

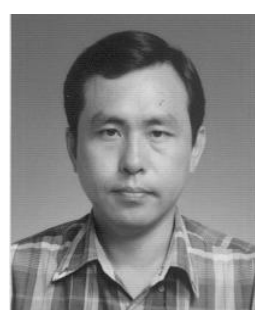

\section{Eung-Seok Kim}

He received his B.S., M.S., and Ph.D. degrees in Electrical Engineering from Hanyang University, Seoul, Korea in 1988, 1990, and 1993, respectively. From 1993 to 1994, he was a Post DOC. in the Control Systems Laboratory of KIST. From 1994 to 1999, he was a Senior Research Engineer in Samsung Advanced Institute of Technology. Since 1999, he has been with Halla University as an Associate Professor in the Department of Control and Instrumentation Engineering. His research interests are advanced control, power electronics, renewable energy system, and energy efficiency control.

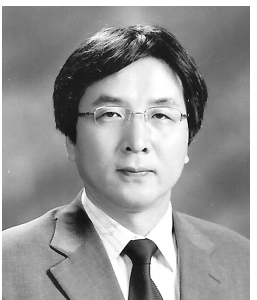

\section{Cherl-Jin Kim}

He received his B.S., M.S., and Ph.D. degrees in Electrical Engineering from Hanyang University, Seoul, Korea from 1980 to 1991. From 1991 to 1995, he was with the Korea Electronics Technology Institute, where he became the Head Researcher of the Control Systems Laboratory. Since 1995, he has been with Halla University as a Professor in the Electrical Engineering Department. His research activities are in the area of power electronics, which includes electrical machine control systems and static converter design fields. 